\title{
Learning fine-grained and category information in navigable real-world space
}

\author{
David H. UtTal \\ Northwestern University, Evanston, Illinois \\ Alinda Friedman \\ University of Alberta, Edmonton, Alberta, Canada \\ AND \\ Linda LiU Hand and Christopher WarRen \\ Northwestern University, Evanston, Illinois
}

\begin{abstract}
Spatial judgments are affected by both fine-grained and categorical knowledge. We investigated whether, and how, the two forms of knowledge are learned in real-world, navigable space, as well as the time course of learning each type of knowledge. Participants were Northwestern University undergraduates who estimated the locations of buildings and other landmarks on campus. The Northwestern campus is roughly divided into three regions whose borders are not easy to discern, either from a map or by navigation. Nevertheless, students often refer to these regions linguistically and use them when making housing decisions, choosing classes, and so forth. We found that knowledge of both the fine-grained configuration of locations and the regional distinctions increased with time. However, regional influences on judgments occurred later in students' time on campus. Consequently, computed distances across the nonexistent border between north and south campus locations became more biased with time. The results have implications for understanding how spatial representations develop in navigable environments.
\end{abstract}

One of the most consistent findings in the past 30 years of spatial cognition research is that mental representations of spatial information often have a categorical structure. Several converging lines of research provide evidence for the psychological reality of categorical representations of spatial information. For example, when asked whether Seattle or Montreal is farther north, people typically respond that Montreal is, even though the opposite is true. People make this error because they respond as if all Canadian cities are north of all U.S. cities, even though this categorical knowledge can lead to large errors (Friedman \& Brown, 2000a, 2000b; Friedman, Kerkman, Brown, Stea, \& Cappello, 2005; Stevens \& Coupe, 1978). Likewise, people consistently overestimate distances that cross imagined or real category boundaries, even if the actual physical distances are equal to or even less than comparable withincategory distances (e.g., Carbon \& Leder, 2005; Friedman \& Montello, 2006; Hirtle \& Jonides, 1985; Hund \& Plumert, 2005; Maki, 1981; McNamara, 1986; Newcombe \& Liben, 1982; Plumert \& Hund, 2001).

Although categorical representations can lead to systematic distortions, they nevertheless serve an important function. For example, in Huttenlocher, Hedges, and Duncan's (1991) category-adjustment model of spatial location judgments, two sources of independent spatial in- formation are combined in a Bayesian fashion to make location estimates from memory (see Cheng, Shettleworth, Huttenlocher, \& Rieser, 2007). One source of knowledge is fine-grained metric information about the location of the items. In Huttenlocher et al.'s (1991) case, the finegrained information is information about the location of dots within the boundaries of a circle; in the case of realworld location estimates, fine-grained information can be the location of cities or other locations at either a global (Friedman, 2009; Friedman \& Brown, 2000a, 2000b) or a navigable (Kitchin \& Fotheringham, 1997) scale.

The second source of spatial information is categorylevel knowledge. This information can also be metric in nature and tends to comprise beliefs about the locations of category boundaries and prototypes. For example, when remembering the locations of dots in a circle, people tend to act as if the circle were divided into quadrants, with the boundaries of the quadrant/categories being (nonexistent) vertical and horizontal lines. Some researchers have suggested that these lines are a cognitive construction (e.g., Huttenlocher et al., 1991), whereas others (e.g., Simmering \& Spencer, 2007; Wenderoth \& van der Zwan, 1991) have suggested that the lines may be caused by features of the visual system and, hence, are experienced as if they had actually been seen. In either case, these category

D. H.Uttal, duttal@northwestern.edu 
boundaries are metrically precise - more so, in the usual case, than the fine-grained location information. When fine-grained knowledge is relatively vague, people rely more heavily on categorical knowledge to make their estimates. The precision of the category boundaries combined with the (usual) vagueness of the memory for a given dot's location leads people to estimate locations that are close to the category's prototype - in this case, the diagonals of the circle. Thus, the combination of item- and categorylevel knowledge leads to biased responses (i.e., responses toward the prototypes). Nevertheless, when the locations of dots in circles are estimated, responses are more accurate, on average, when the prototype is used than they would be if category knowledge did not play a role in the estimates and if participants relied only on (vaguer) finegrained location knowledge. However, in real-world location estimates, the category boundaries may also be precise (e.g., political borders), but, from a metric standpoint, they can be mislocated by large amounts (e.g., people may believe that a given region is too far north or south of its actual location; Friedman \& Brown, 2000a; Friedman \& Montello, 2006).

The present research was designed to address two questions that follow from using the category-adjustment model to make predictions about the representation and use of fine-grained and category-level information in location estimates. The first concerns whether, and to what extent, models of the representation of item- and category-level information hold in larger, navigable spaces. The second concerns the time-course during which both levels of information are learned; for example, do both fine-grained and categorical knowledge develop simultaneously, or is one learned before the other?

With respect to the first question, most research in this field has involved relatively small-scale spaces, such as a computer screen (e.g., Huttenlocher, Hedges, Corrigan, \& Crawford, 2004; Newcombe \& Chiang, 2007), a sandbox (Huttenlocher, Newcombe, \& Sandberg, 1994), a scale model (e.g., DeLoache, 1989, 2000; Hund \& Plumert, 2002, 2003, 2005), or a small room (e.g., Hermer \& Spelke, 1994, 1996; McNamara, Rump, \& Werner, 2003; Mou, McNamara, Valiquette, \& Rump, 2004). Other research has been conducted with respect to large-scale global spaces that are not typically navigable (e.g., the countries in North America; Friedman \& Brown, 2000a, 2000b; Friedman et al., 2005; Friedman \& Montello, 2006). Relatively few studies about the representation and use of fine-grained and category knowledge have been done in spaces that are navigable and, thus, can be learned through many different means (e.g., walking, public transport, automobile, maps, directions from others). Indeed, a general criticism of much psychological research that tests the category-adjustment model - and perhaps research in spatial cognition more generally-is that it has focused on scales that are so small that they render the findings potentially ungeneralizable, even to slightly larger spaces (Liben, 2003; Montello, 1993). Even modest changes in the size of a space can dramatically alter how spatial information is represented mentally (e.g., Acredolo, 1981; Learmonth, Newcombe, Sheridan, \& Jones, 2008).
As noted above, for small-scale spaces (e.g., the size of a computer screen; Huttenlocher et al., 1991), the combination of both category- and fine-grained information leads to optimal judgments. For larger, geographic-scale spaces, category knowledge has been shown to be detrimental to overall accuracy, primarily because people's beliefs about the locations of category boundaries are "off" by a comparatively large amount. For example, Friedman et al. (2005) showed that Canadian, American, and Mexican participants all placed most Mexican cities at or near the equator; presumably, this global landmark serves the same function for all participants and causes their estimates to be approximately 1,500 miles too far to the south (see also Friedman, 2009). Nevertheless, all three groups of participants were relatively accurate in placing the cities within their home regions in their correct northto-south relations, and the relations were metrically (and strongly) linear. This finding is an example of how the fine-grained knowledge can be accurate within regions, but, when this fine-grained knowledge is combined with category knowledge, the overall result is highly inaccurate (Friedman, 2009; Friedman \& Montello, 2006). Here, we tested whether category-level knowledge at the navigable scale can also lead to inaccurate performance. If so, this finding would be a further constraint on the categoryadjustment model.

Our second goal was to examine how fine-grained and category-level information is acquired over the course of one or more years in a large-scale, navigable space: the campus of Northwestern University in Evanston, Illinois. We measured location estimates of buildings on the Northwestern campus from first-year students during the first three quarters of the academic year, and from secondthrough fourth-year students during the same time period, using a cross-sectional design for both groups. This design allowed us to examine the acquisition of both fine-grained and category knowledge and to determine, for example, whether their learning overlaps.

The layout of the Northwestern University campus is a particularly interesting test bed for studying how finegrained and category-level information are represented and used in navigable spaces. Originally, the northern and southern sections of the campus were clearly defined areas and were separated by substantial empty space. However, the campus has filled in, and there is no longer a clear geographic boundary separating the northern and southern sections of campus. A third, midcampus, area also has emerged. For decades, it has not been possible to look at a map of the campus and be sure which buildings are on which section of campus (see Figure 1). Nevertheless, students often refer to buildings as being "up north" or "down south." In addition, the three campus areas now house different groups of buildings by academic discipline; for example, science and technology are on the north campus, and music and fine arts are on the south campus. Therefore, there are probably both linguistic and cultural bases for a cognitive division of the campus. Indeed, although the north and south campus do correspond to general geographic areas, they are now as much a cognitive construction as a geographic reality. At the same time, the 


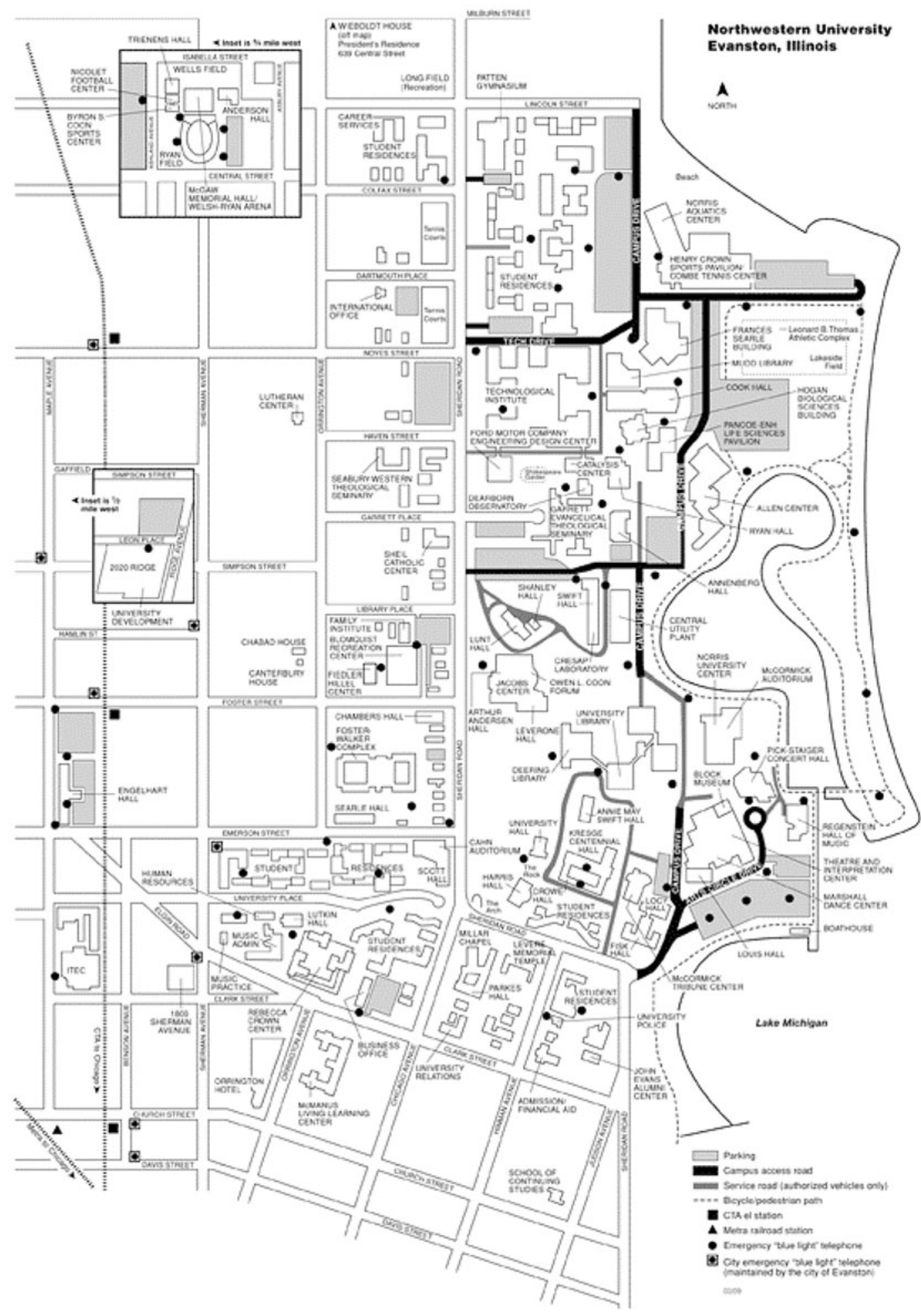

Figure 1. Standard map of the Evanston campus of Northwestern University, available at www.northwestern .edu/campus-life/evanston-campus.

campus is also small enough and course requirements are broad enough, that it is reasonable to assume that students will have navigated the entire campus during their tenure at the university and that they can be expected to acquire at least some information about locations on both north and south campus. We focus on north and south campus, because linguistic references to these areas are much more common than are references to midcampus. 
We made two sets of predictions regarding the representation and learning of campus locations. The first set concerns the learning of fine-grained information, and the second concerns the learning of category-level information. We predicted that fine-grained locational knowledge would improve, certainly for first-year students and possibly across the course of 4 years on campus. Consequently, people should exhibit improved knowledge for the configuration among all the buildings on campus (i.e., building-to-building relational and metric knowledge among items). The second set of predictions concerned the acquisition of category-level knowledge, which we hypothesized should also develop with time on campus. We discuss each of these in turn below. Notably, a key aspect of our design is that it allowed us to determine whether fine-grained and category-level information are learned at the same rate. To our knowledge, this is the first time that this type of separation in rate of spatial learning as a function of type of information over a long period of time has been examined.

We examined the accuracy of fine-grained knowledge in three ways. First, we analyzed the $x$ - and $y$-dimensions independently. Whereas the match between the estimates and configuration of the campus as a whole is important because of theoretical interest in how object-to-object locations are learned during navigation (e.g., Mou et al., 2004; Waller, Montello, Richardson, \& Hegarty, 2002), from the perspective of the category-adjustment model, we had specific predictions about the north-south dimension. In particular, because of the cognitive "construction" of the north and south campus, we hypothesized that knowledge about buildings on the $y$ - (north-south) dimension would underlie any observed effects of category knowledge. Moreover, if the cognitive and linguistic conventions used to refer to the buildings or landmarks on campus play a role in their represented locations, then the north-south locations of the buildings and landmarks should be learned earlier than the east-west locations. If so, the Pearson correlations between actual and estimated locations on the $y$-dimension should be stronger earlier in a participant's experience on campus than the correlations on the $x$-dimension would be. We used Pearson correlations, because we wanted to examine the metric accuracy of the acquired knowledge.

The second way in which we examined the accuracy of fine-grained knowledge was to analyze the data with a bidimensional regression (BDR). This type of analysis quantifies the accuracy of knowledge about the overall configuration of buildings (Friedman \& Kohler, 2003; Tobler, 1965, 1994). Briefly, BDR is a statistical method that computes a correlation between two or more twodimensional Euclidean configurations of locations and provides parameters that describe the extent to which an individual's cognitive map must be rotated, translated north-south and/or east-west, and scaled to fit into an actual map and vice versa. In other words, it is a twodimensional "best fit" among a set of points. Thus, just as a Pearson $r$ reflects the magnitude of the linear relation between two unidimensional variables, the magnitude of the
BDR coefficients for each participant reflects how much variance in the configuration of the estimated values of a bidimensional variable is accounted for by the actual configuration of locations in Euclidean space (or vice versa; see Friedman \& Kohler, 2003, for details). The BDR coefficients inform us about the growth of fine-grained (parametric) configurational knowledge about the campus as a whole, and thus, they address the issue of how object-toobject relations are learned through navigation. We had no a priori predictions about the remaining parameters of the BDR analysis, and some of them (e.g., the north-south and east-west translation parameters) could plausibly be part of both fine-grained and category-level knowledge, so the analyses of these parameters (and further explanation) are given in the Appendix.

The third measure of fine-grained knowledge was the raw accuracy of participants' placements, computed as the absolute error in pixels for each estimate for each building, separately for the north-south and east-west dimensions (i.e., |estimated value - actual value|). We used absolute errors on each dimension, instead of signed errors, because signed errors can give a false impression of accuracy. If participants are uncertain about relative positions on a dimension, their positive errors will cancel their negative errors. Absolute errors, in contrast, are a measure of this uncertainty. In general, absolute errors reveal different information than correlations do. For example, in Friedman (2009), within-region correlations between actual and estimated locations in familiar regions were relatively good, but absolute errors for the same locations were quite large. This result indicated that the withinregion fine-grained location information was metrically accurate, but the items were inheriting inaccurate absolute location information from the regional level. In the present case, it should be noted that because the campus is rectangular, with the north-south dimension being approximately 1.4 times as large as the east-west dimension, there is less absolute "room" for east-west errors than for north-south errors. Consequently, it is possible that absolute errors on the east-west dimension could be less than those for the north-south dimension, because there is less physical room to respond to in the former direction than in the latter. However, if categorical information facilitates the acquisition of correct relative north-south locations between buildings, absolute errors to east-west locations might actually be greater than north-south errors are.

Our second set of predictions concerned the learning of categorical knowledge about the campus. Even as buildingto-building knowledge on the north-south dimension and configurational knowledge may improve with time, it is still possible that the buildings in the north of campus are drawn apart from the buildings in the south, leading to the representational underpinnings of any observed categorylevel effects. This should happen if categorical beliefs about the north and south campus develop with time and are influential in the representation of campus locations. Alternatively, a psychological "border" between north and south campus might develop with time. In either case, the implication is that distances computed from location es- 
timates of buildings within the north or within the south campus should be less than physically equal distances computed from location estimates of buildings across the invisible north-south boundary. This type of "border effect" has been demonstrated repeatedly. For example, Newcomb and Liben (1982) found that children consistently overestimated distances that traversed boundaries, relative to comparable distances that did not. Maki (1981) found similar results for judgments of distances that either did or did not traverse state borders. Borders can influence judgments of geographic distance, even when the physical borders no longer exist and are, therefore, necessarily psychological (e.g., the former Berlin Wall; see Carbon \& Leder, 2005).

We examined the influence of categorical knowledge by computing the Euclidean distance between pairs of buildings in which both members of a pair were located in either the northern or the southern region of the campus (within-region pairs) or between pairs in which one was located in the north and the other was located in the south (between-region pairs). The pairs were selected so that the average within-category Euclidean distances were equated to the between-category distances to within 1 pixel. The average within- and between-region computed distances were then analyzed to determine whether there were border effects and, if so, when they arose. If category knowledge increases with time, the between-category estimates should eventually become larger than within-category estimates (e.g., Friedman \& Montello, 2006; Maki, 1981).

In summary, then, we have a set of somewhat counterintuitive predictions: At the same time that configural knowledge is improving, the computed Euclidean distance between estimated locations should show an increasing tendency for the categories to separate. Demonstrating that both fine-grained accuracy and category-level bias increase with time - but at different rates - would provide strong evidence for the independence of these two types of knowledge. This pattern of results would provide evidence for the category-adjustment model but would constrain that model by showing that, at a navigable scale (as at a global scale), the categorical information causes inaccuracies in the representation, rather than making it more accurate.

The design of our study also allowed us to address an additional issue that could affect participants' estimation of locations: the influences of physical boundaries on participants' spatial judgments. One group of participants made their responses on a "blank slate": a computer screen that was empty, except for the location of a central campus building. A second group had precise east and west boundaries (the Chicago Transit Authority L train tracks on the west and the border of Lake Michigan on the east). Kitchin and Fotheringham (1997), who also investigated location estimates in a navigable space, found that including precise boundaries greatly improved estimation accuracy. In the present study, we naturally expected that the groups that had cues would be more accurate than those that did not (see Simmering \& Spencer, 2007). Nevertheless, the linguistic and physical categories are references that highlight the north-south dimension. Thus, the addition of east-west border information should not affect any influence of categorical information in the north-south dimension, and biases in that dimension should still be evident.

\section{METHOD}

\section{Participants}

All 162 participants were full-time, undergraduate students on the Evanston Campus of Northwestern University. Most were enrolled in introductory psychology and received credit for the research participation requirement of that course. Because most of the students in this introductory class were first- and second-year students, additional third- and fourth-year participants were recruited during the summer and were compensated $\$ 5$ for their time.

Because we wanted to roughly balance for residency on north and south campus, all participants completed a brief, preliminary survey, in which they indicated where they lived on campus currently and during the past year. Indeed, because only 8 participants were recruited who lived on midcampus, their data were excluded from further consideration. In addition, a total of 12 participants began the experiment, but either they did not complete it or their data were not included in the final analyses ( 5 were excluded because of computer error, 5 were excluded because they did not indicate where they lived on campus or they lived far from campus, 1 was excluded because her data on some measures exceeded 3 SDs from the mean of the other participants, and 1 was excluded because he was the only participant among the first-year students who was in the fourth quarter - that is, attending summer school). Thus, in the final data set, there were 142 participants: 93 first-year students (36 males, 57 females; mean age 18.4 years; range $17-20$ years; 3 participants did not give their ages; 36 students were in their first quarter, 30 were in their second, and 27 were in their third); 24 second-year students (12 males, 12 females; mean age 19.3 years; range $18-20$ years); 13 third-year students ( 8 males, 5 females; mean age 20.6 years; range 20-21 years); and 12 fourth-year students ( 7 males, $5 \mathrm{fe}-$ males; mean age 21.6 years; range $21-22$ years). In total, there were 49 upper-level students (19 males, 22 females; mean age 20.2 years, range $18-22$ years).

\section{Stimuli}

Selection of landmarks. Figure 1 is a map of the campus that is freely available on-line (www.northwestern.edu/campus-life/ evanston-campus/) and is included in recruitment and orientation materials for students. It can be seen that there are no natural or manmade borders that would formally demarcate the campus into regions. Figure 2 shows the stimulus map that was seen by participants in the cue group.

To assign buildings to regions, we divided the campus into three sections - south, middle, and north — on the basis of the following criteria: South campus was defined as beginning at CVS Pharmacy and ending at the south end of an imaginary line extending Emerson Street to Lake Michigan; midcampus was from the north end of this imaginary line to an imaginary line extending Campus Drive through Lake Michigan; and north campus was from an imaginary extension of Campus Drive to the north end of Lincoln Street.

The psychological divisions were confirmed with a questionnaire filled out by 12 additional participants, who were familiar with the campus. Participants were given a list of the campus buildings and were asked to indicate whether each of the buildings was in south, middle, or north campus. Overall agreement with our assignment of the buildings was $93.4 \%$. Agreement was $100 \%$ for 15 of the 28 buildings. Agreement for the north and south campus buildings was $96.5 \%$ and $98.5 \%$, respectively. Agreement was low for only 1 building - Swift Hall — with 4 participants indicating that it was in midcampus, with 6 indicating that it was in north campus, and with 


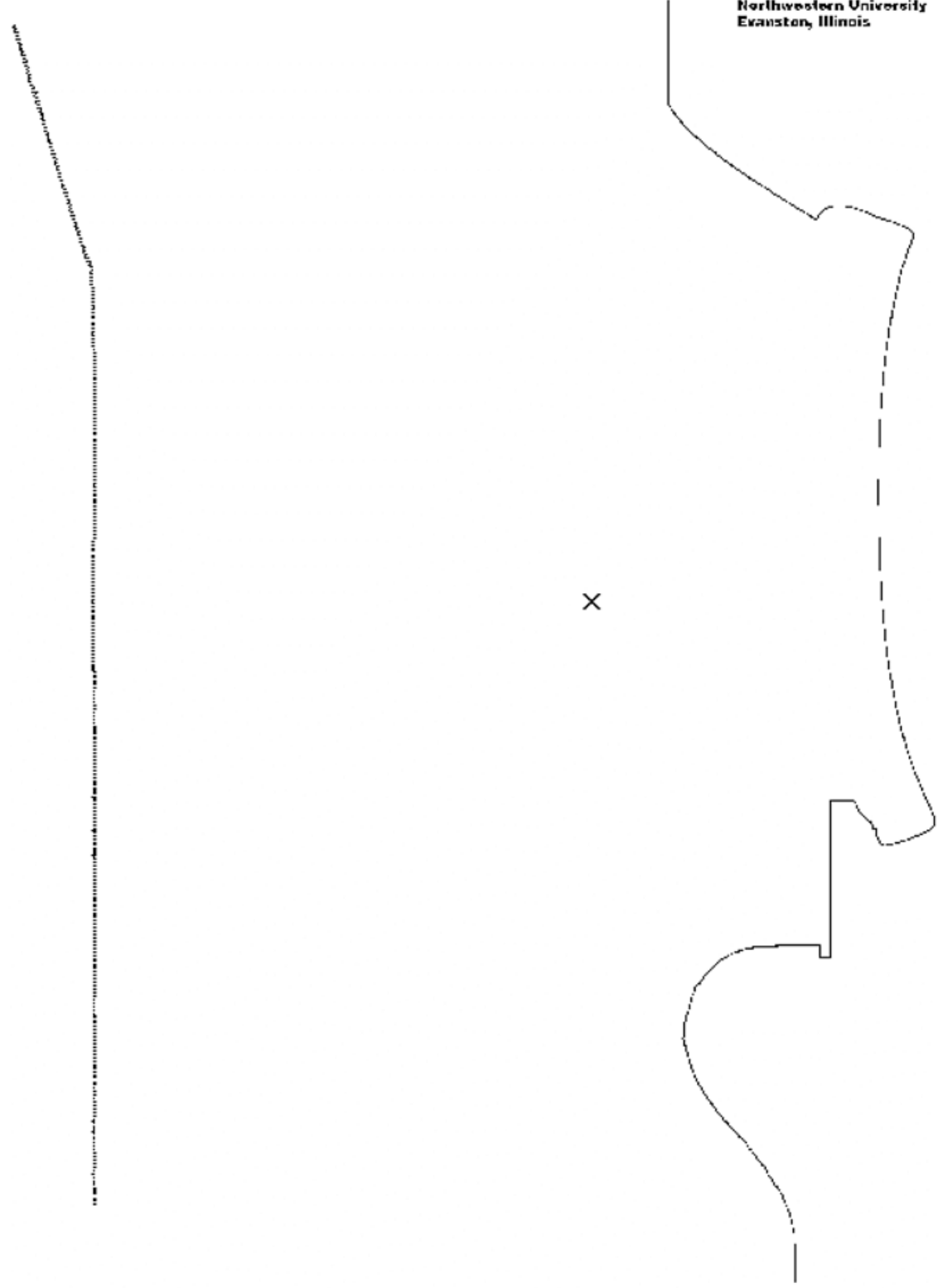

Figure 2. The response screen for the cue group. The lines were not present on the no-cue response screen, which showed only the single $X$ and the Northwestern University label.

2 indicating that it was in south campus. Part of the confusion may stem from the fact that there are two buildings on campus with the name Swift: Swift Hall and Annie Mae Swift Hall. Note that Swift Hall was not used as one of the target stimuli; it was the one building whose location was indicated on the response screens for both the cue and no-cue groups. Nevertheless, these results confirmed our division of the campus into three regions and show that there is good agreement among students regarding which buildings are in which regions.

Within each region, the target locations included the main entrances of major campus buildings and other physical landmarks that were judged by pilot testing to be the most salient among the student body (see Figure 1). We selected these stimuli so that they were well distributed throughout all three regions, for a total of 11 north campus locations, 6 midcampus locations, and 11 south campus locations. There were unequal numbers of buildings per region, because there are fewer buildings in midcampus, due to several preserved green spaces.

\section{Procedure}

We tested all participants in Swift Hall, which is located very close to the geographic center of campus. After providing informed consent, participants were asked to rate their knowledge of each of 28 campus locations on a scale of $0-9$. We provided examples for most of the points on the scale; for example, 1 or 2 was defined as a place that the participant had heard of but never visited, and an 8 or 9 was defined as a location about which the participant knew quite a bit. We gave knowledge ratings primarily as a means of familiarizing participants with the set of places they would be estimating in the subsequent phase of the task (see Friedman \& Brown, 2000a). The target locations were presented in a random order for each participant. 
Location recall. Next, we assessed participants' recall of the target locations. All participants recalled the locations one at a time by "dragging and dropping" an X that stood for a particular target place to the location on a computer screen where they thought that particular target should be located.

On each trial, the name of the target location appeared at the top of the screen, and an X appeared at the bottom of the screen. Participants were told to use the mouse to drag the $\mathrm{X}$ to show where the building whose name appeared at the top of the screen was located on campus. They were instructed to move the $\mathrm{X}$ until the middle of the $\mathrm{X}$ was exactly where they thought the building (or other landmark) was and to press a mouse button to drop the $\mathrm{X}$ at that location. When participants were satisfied with their response, they pressed the Enter key. E-Prime software (Psychology Software Tools, Inc.) presented the stimuli and recorded the estimated locations in pixel units.

The procedure was repeated for each location individually. Participants placed one location at a time, and the only building that appeared on all screens for all participants was Swift Hall, the location at which they were being tested. Locations were presented in a different random order for each participant.

Participants were assigned randomly to either the cues or no-cues condition. The cues condition response screen included lines representing the elevated train tracks (the west boundary of the campus) on the left side of the screen and a line representing Lake Michigan (the east boundary of campus) on the right side of the screen, in addition to the X demarking Swift Hall (see Figure 2). Participants in the no-cues condition responded on a screen that depicted only the $\mathrm{X}$ representing Swift Hall.

\section{Data Analysis}

The location estimate for each target stimulus was derived from the cursor position that each participant clicked on for each building; that position provided the estimated $x, y$ pixel values. We used these location estimates to examine the acquisition of, and changes in, both fine-grained and category-level knowledge.

We analyzed the data from the first-year students alone to see whether both fine-grained configural and category knowledge increased over the course of their three quarters on campus. We then analyzed the data from the upper-level students to see whether configural and categorical knowledge continued to increase. The data from both groups were then combined to examine the influence of overall time on campus on both fine-grained configural knowledge and category effects. For all analyses reported, we adopted a $p<.05$ alpha level and use $\eta_{\mathrm{p}}^{2}$ as the measure of effect size.

Because parametric correlations are not normally distributed (as $r$ increases, the sampling distributions become skewed), we transformed all correlations (Pearson and bidimensional) using Fisher's $r$-to- $z$ method before they were submitted to statistical tests. The reported correlations were computed by back-transforming the mean of each $r$-to-z-transformed correlation.

\section{RESULTS}

\section{Knowledge Ratings}

In a cue group $\times$ quarter ANOVA, first-year students reported increasing knowledge of the locations as a function of quarter; the means for the first, second, and third quarters were 5.23, 5.43, and 5.88, respectively, but the effect only approached significance $\left[F(2,87)=2.56, M S_{\mathrm{e}}=\right.$ $\left.1.35, \eta_{\mathrm{p}}^{2}=.056, p=.085\right]$. No other effects were significant. The class (first year vs. upper level) $\times$ cue group (cues vs. no cues) ANOVA revealed only a main effect of class $\left[F(1,138)=12.21, M S_{\mathrm{e}}=1.40, \eta_{\mathrm{p}}^{2}=.081\right]$. The mean for the first-year students was 5.49, and for the upper-level students it was 6.23. Thus, self-reported knowledge continued to increase with time on campus after the first year.

\section{Fine-Grained Accuracy}

As noted above, we examined fine-grained accuracy with three measures: the linear relation between the 28 actual and estimated locations for the $x$ - and $y$-dimensions separately, using Pearson correlations, the Euclidean configural accuracy among all the locations measured using BDR coefficients, and absolute accuracy using absolute distances (in pixel units) between actual and estimated locations for each dimension separately.

For the first-year students, we submitted the Fishertransformed Pearson correlations to a cue group (cues, no cues $) \times$ quarter $(1-3) \times$ dimension $(x, y)$ mixed ANOVA, in which dimension was the repeated measure. There was a main effect of cue group $\left[F(1,87)=4.93, M S_{\mathrm{e}}=0.53\right.$, $\left.\eta_{\mathrm{p}}^{2}=.054\right]$, a main effect of quarter $[F(1,87)=15.13$, $\left.M S_{\mathrm{e}}=0.54, \eta_{\mathrm{p}}^{2}=.258\right]$, and a cue group $\times$ quarter interaction $\left[F(1,87)=3.54, M S_{\mathrm{e}}=0.54, \eta_{\mathrm{p}}^{2}=.075\right]$. Importantly, there was also a main effect of dimension $\left[F(1,87)=70.54, M S_{\mathrm{e}}=0.16, \eta_{\mathrm{p}}^{2}=.448\right]$, and dimension did not interact with any other factor for the firstyear students. The data across all four factors are shown in Figure 3.

The correlations were generally higher for the cue group than for the no-cue group. In addition, within each cue group and for all three quarters, correlations on the $y$-dimension were always stronger than those on the $x$-dimension. Correlations were stronger on the

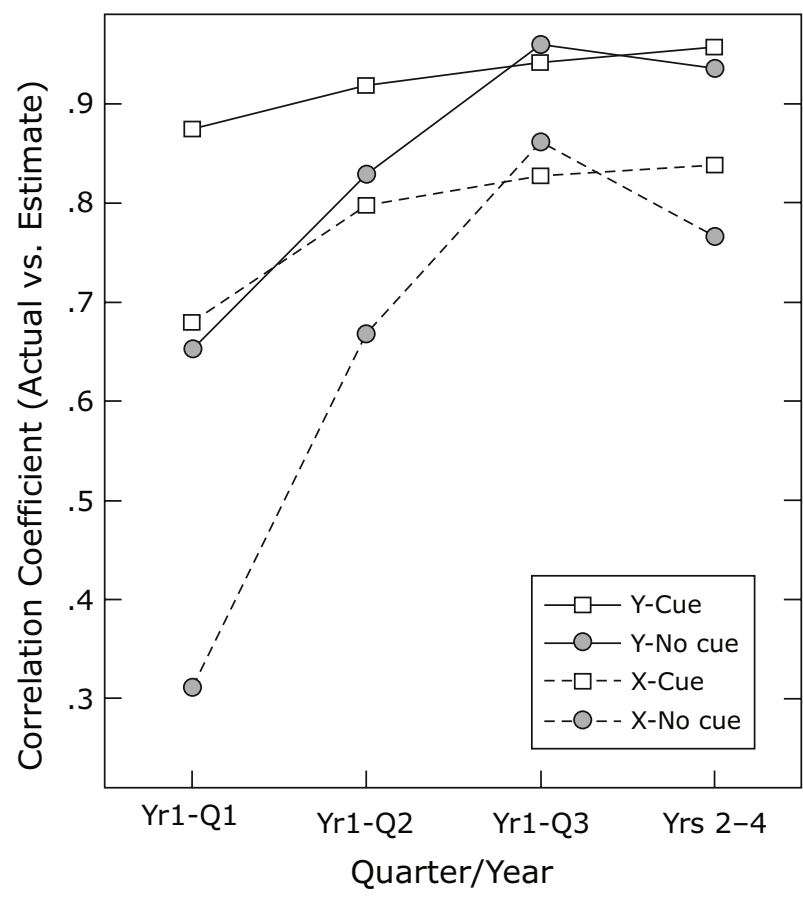

Figure 3. Pearson correlations between estimated and actual locations as a function of cue group, quarter or year, and dimension $(x$ or $y)$. Because dimension was within subjects, it was not obvious what to use as error bars in this situation. Across classes, the $95 \%$ confidence limits for the $x$ dimension were $.493-.918$ for the cue group and .288-.853 for the no-cue group. For the $y$ dimension, these limits were $.792-.972$ for the cue group and $.637-.940$ for the no-cue group. 
$y$-dimension than on the $x$-dimension for the first quarter $\left[F(1,87)=20.13, M S_{\mathrm{e}}=0.22, \eta_{\mathrm{p}}^{2}=.359\right]$, indicating that the north-south locations of buildings were learned very rapidly indeed.

For second- through fourth-year students, only the main effect of dimension was significant $[F(1,47)=102.44$, $\left.M S_{\mathrm{e}}=0.11, \eta_{\mathrm{p}}^{2}=.685\right]$; the back-transformed means were .521 and .791 for the $x$ - and $y$-dimensions, respectively (see Figure 1). Importantly, in the ANOVA that included class, cue group, and dimension as factors, all three main effects were significant [class, $F(1,138)=10.39, M S_{\mathrm{e}}=$ $0.65, \eta_{\mathrm{p}}^{2}=.070$; cue group, $F(1,138)=5.98, M S_{\mathrm{e}}=0.65$, $\eta_{\mathrm{p}}^{2}=.042$; dimension, $F(1,138)=155.34, M S_{\mathrm{e}}=0.14$, $\left.\eta_{\mathrm{p}}^{2}=.530\right]$, as was the interaction between class and dimension $\left[F(1,138)=3.89, M S_{\mathrm{e}}=0.14, \eta_{\mathrm{p}}^{2}=.027\right]$. No other interactions were significant $(F \mathrm{~s}<1)$. Across quarters for the first-year students, the mean correlations for the $x$ - and $y$-dimensions were .707 and .882 , respectively; for the second- through fourth-year students, they were .806 and .947 . The data in Figure 3 suggest that, by the end of the first year, the north-south and east-west locations of the campus buildings were relatively well learned, and the relations were strongly linear, although performance was better for the group that had cues than for the group that did not. In addition, estimates on the north-south dimension remained high through the later years on campus, yet there was still a significant difference between the two dimensions.

In sum, the correlational data show that both within the first year and across the 4 years, fine-grained metric accuracy about the north-south and east-west locations of the target buildings increased, but the north-south correlations began at a higher level than the east-west ones did. Notably, the correlations for the first-year students in their third quarter were quite similar to the correlations for the upper-level students. Consequently we believe that most of the learning of fine-grained locations occurred during the course of the first year on campus.

To examine learning of configural knowledge among the buildings, a BDR coefficient was determined from each individual's 28 estimates; the actual campus locations (in $x, y$ pixel units) were the independent variable, and the estimated locations (also in pixels) were the dependent variable. For first-year students, we analyzed the resulting Fisher-transformed regression coefficients in the same way as the correlations (but without dimension as a factor, because the BDR analysis is already two-dimensional). First-year students' accuracy was affected by the presence of cues $\left[F(1,87)=10.83, M S_{\mathrm{e}}=0.16, \eta_{\mathrm{p}}^{2}=.111\right]$. Fine-grained configural knowledge was higher for participants who had perceptual cues versus those who did not; the back-transformed mean regression coefficients were .883 and .805 , respectively. There was also a main effect of quarter $\left[F(2,87)=13.73, M S_{\mathrm{e}}=0.16, \eta_{\mathrm{p}}^{2}=.240\right]$. The back-transformed means for the first, second, and third quarters were $.754, .852$, and .906 , respectively.

For upper-level students, the effect of cue was also significant $\left[F(1,47)=6.91, M S_{\mathrm{e}}=0.20, \eta_{\mathrm{p}}^{2}=.128\right]$. The back-transformed means were .941 for the cue group and .885 for the no-cue group. In the combined analysis of all students, there were significant main effects of cue $\left[F(1,138)=16.13, M S_{\mathrm{e}}=0.20, \eta_{\mathrm{p}}^{2}=.105\right]$ and, importantly, of class $\left[F(1,138)=19.47, M S_{\mathrm{e}}=0.20, \eta_{\mathrm{p}}^{2}=.124\right]$. The effect of class indicated that, on average, configural knowledge of the campus continued to improve after the first year; the back-transformed means for the first-year and upper-level students were .839 and .917 , respectively. However, the BDR coefficient for the first-year students in their third quarter was similar to that of the upper-level students (.906 and .917 , respectively). Thus, it appears as if fine-grained configural knowledge was acquired primarily through the first year.

The third way we examined fine-grained accuracy was by analyzing the absolute errors in pixel units of distance, independently for the $x$ - and $y$-dimensions. The first-year students had significant main effects of cue group $\left[F(1,87)=32.69, M S_{\mathrm{e}}=21,244.91, \eta_{\mathrm{p}}^{2}=.273\right]$ and quarter $\left[F(2,87)=10.16, M S_{\mathrm{e}}=21,244.91, \eta_{\mathrm{p}}^{2}=\right.$ $.189]$, and there was an interaction between the two variables $\left[F(2,87)=3.56, M S_{\mathrm{e}}=21,244.91, \eta_{\mathrm{p}}^{2}=.076\right]$. There was also a nearly significant effect of dimension $\left[F(1,87)=3.53, M S_{\mathrm{e}}=11,610.24, \eta_{\mathrm{p}}^{2}=.039\right]$ and a dimension $\times$ cue group interaction $[F(1,87)=15.02$, $\left.M S_{\mathrm{e}}=11,610.24, \eta_{\mathrm{p}}^{2}=.147\right]$. No other effects were significant. The data are shown in Figure 4. It is clear that the cue group was more accurate than the no-cue group on both dimensions and that there was a significant decline in absolute error across quarters. The cue $\times$ dimension interaction indicated that, for the no-cue group, the north-south dimension was more accurate than the east-west dimension was, whereas for the cue group, the

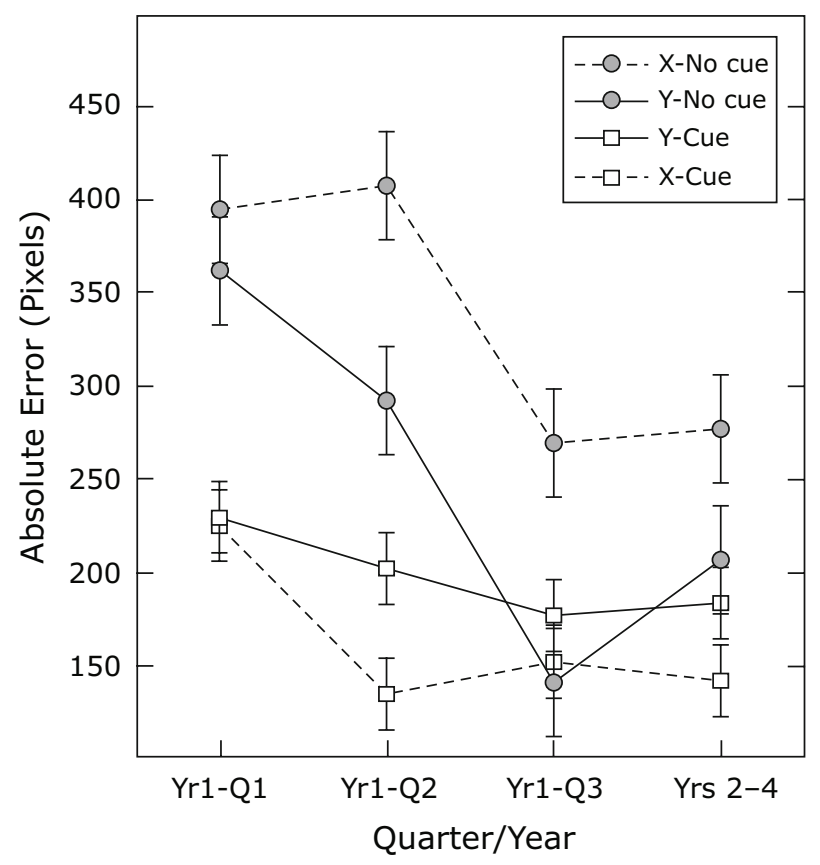

Figure 4. Absolute errors between estimated and actual locations as a function of cue group, quarter or year, and dimension $(x$ or $y)$. Error bars are $95 \%$ confidence intervals computed across all participants (first year and upper level) but are computed separately by cue group (see Loftus \& Masson, 1994). 
opposite was true. This interaction probably stems from the fact that the lines constrain the placements for the cue group, and there was less room for error in the east-west dimension when there were cues. In addition, there was not much difference between the dimensions in general for the cue group. But it is important to note that accuracy was higher in the north-south dimension when there were no cue constraints. This result indicates (again) that participants were more familiar with the locations of the buildings on this dimension than they were on the eastwest dimension.

For the upper-level students, there was also a main effect of cue group $\left[F(1,47)=5.25, M S_{\mathrm{e}}=28,092.17, \eta_{\mathrm{p}}^{2}=\right.$ $.100]$ and a dimension $\times$ cue group interaction $[F(1,47)=$ $\left.9.14, M S_{\mathrm{e}}=8,148.17, \eta_{\mathrm{p}}^{2}=.163\right]$, which took the same form as that for the first-year students (see Figure 4).

In the analysis that included both the first-year and upper-level students, importantly, the main effect of class was significant; upper-level students were more accurate, on average, than first-year students $[F(1,138)=$ 7.17, $\left.M S_{\mathrm{e}}=26,947.80, \eta_{\mathrm{p}}^{2}=.049\right]$. In addition, there was, once again, a main effect of cue group $[F(1,138)=$ $\left.25.96, M S_{\mathrm{e}}=26,947.80, \eta_{\mathrm{p}}^{2}=.158\right]$ and a dimension $\times$ cue group interaction $\left[F(138)=19.18, M S_{\mathrm{e}}=10,518.76\right.$, $\left.\eta_{\mathrm{p}}^{2}=.122\right]$ but no other significant effects.

In sum, all three measures of fine-grained knowledge converge on roughly the same conclusions. First, cues clearly contributed to accurate performance. Second, because the cues constrained the east-west, but not the north-south, estimates, the former were more accurate than the latter at an absolute level, but not in terms of relational knowledge (for either the separate dimensions or the configuration as a whole). Third, fine-grained knowledge was gained with time on each dimension alone and on the configuration of buildings as a whole. Finally, the Pearson correlations showed that the parametric relation between north-south locations was stronger than it was for east-west locations and at an earlier stage in the participants' time on campus.

\section{Categorical Accuracy}

We measured the influence of categorical information on the estimates, using the computed Euclidean estimated distances between 12 preselected pairs of buildings that were both in the north campus ( $n=3$ pairs), both in the south campus ( $n=3$ pairs), or between categories $(n=$ 6 pairs). The actual distances between the pairs in the north, south, or between categories were 678,679 , and 677 pixels, respectively. For comparison, each pixel was equivalent in distance to approximately $1.092 \mathrm{~m}$ on the campus, so a distance of 678 pixels would equal approximately $750 \mathrm{~m}$.

For the first-year students, in an ANOVA in which cue and quarter were between subjects and category type (within- vs. between-category pairs) was within subjects, there was a main effect of cue group $[F(1,87)=25.91$, $\left.M S_{\mathrm{e}}=105,190.46, \eta_{\mathrm{p}}^{2}=.229\right]$. In general, the computed distances for the no-cue group were larger ( 960 pixels) than those for the cue group (714 pixels). There were also interactions between cue group and quarter $[F(2,87)=4.63$,
$\left.M S_{\mathrm{e}}=105,190.46, \eta_{\mathrm{p}}^{2}=.096\right]$ and between cue group and category type $\left[F(1,87)=5.06, M S_{\mathrm{e}}=54,899.86, \eta_{\mathrm{p}}^{2}=\right.$ $.055]$. The cue $\times$ quarter interaction showed again that the perceptual cues constrained the overall distances but that, when there were no cues, the computed within- and between-category distances generally increased with time. The distances for the first, second, and third quarters for the cue group were 727,652 , and 763 pixels, respectively, whereas those for the no-cue group were 807, 1,082, and 993 pixels. For the cue group $\times$ category type interaction, there was not a consistent pattern (the means for the withinvs. the between-category pairs for the cue group were 690 and 748 pixels, respectively; those for the no-cue group were 1,015 and 906 pixels). There is, therefore, a hint that the cues resulted in a category effect, but the absence of cues resulted in an opposite pattern. Thus, it appears that first-year students were acquiring primarily fine-grained configural knowledge. For upper-level students, there was again a main effect of cue group $\left[F(1,47)=9.12, M S_{\mathrm{e}}=\right.$ $\left.71,052.94, \eta_{\mathrm{p}}^{2}=.163\right]$. The no-cue group estimated larger distances between buildings than did the cue group ( $890 \mathrm{vs}$. 725 pixels, respectively). Importantly, there was an effect of category type that approached significance $[F(1,47)=$ $\left.4.03, M S_{\mathrm{e}}=34,297.94, \eta_{\mathrm{p}}^{2}=.079, p=.051\right]$. As we expected, the between-category computed distances were larger than the within-category estimates ( 839 vs. 745 pixels, respectively). In addition, there was a cue $\times$ category type interaction $\left[F(1,47)=5.95, M S_{\mathrm{e}}=34,297.94, \eta_{\mathrm{p}}^{2}=\right.$ .112]. Interestingly, there was a relatively large category effect when upper-level students had perceptual cues (640 vs. 809 pixels for within- vs. between-category distances, respectively). However, when there were no cues, the computed distances were roughly equivalent ( 898 vs. 882 pixels for within- vs. between-category distances). Apparently, the cues allowed the senior students to better express their categorical knowledge (and thus, to be less accurate in the distances computed for their estimates). Recall that the actual distances averaged 678 pixels for both within- and between-category pairs. Thus, the cues allowed the upper-level students to be accurate within categories but, at the same time, to spread the buildings out from the center, thus, causing the between-category estimates to increase.

In the ANOVA that combined classes, there was a main effect of cue group $\left[F(1,138)=24.67, M S_{\mathrm{e}}=\right.$ $\left.101,639.94, \eta_{\mathrm{p}}^{2}=.152\right]$ and a cue $\times$ category type interaction $\left[F(1,138)=8.14, M S_{\mathrm{e}}=48,544.81, \eta_{\mathrm{p}}^{2}=.056\right]$. Importantly, the class $\times$ category type interaction was significant $\left[F(1,138)=3.91, M S_{\mathrm{e}}=48,544.81, \eta_{\mathrm{p}}^{2}=\right.$ $.028]$. The interaction among class, category type, and cue group is shown in Figure 5. For first-year students, there were no differences between within- and betweencategory distances ( 853 vs. 820 pixels, respectively), but for the second- through fourth-year students, there was a significant difference (769 vs. 845 pixels). Thus, overall, configural knowledge was expressed before category knowledge: The north-south and BDR regression coefficients for the first-year students increased throughout the three quarters of their first year, but they did not exhibit strong category effects. In contrast, the upper-level stu- 


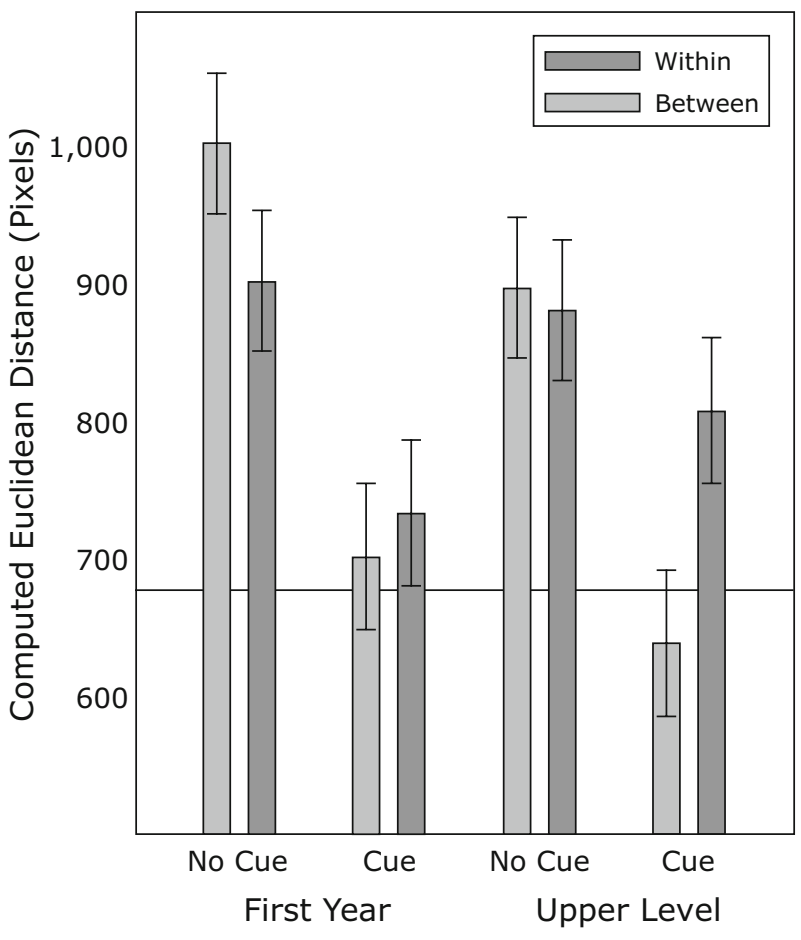

Figure 5. Estimated distances computed from three pairs of locations within each of the northern and southern regions and from six pairs of locations between regions as a function of cue group and class. The horizontal line is the average actual distance between building pairs for both within- and between-category pairs. Error bars are $95 \%$ confidence intervals computed across all participants (first year and upper level), but are computed separately by cue group (see Loftus \& Masson, 1994).

dents showed an effect of category-level information in the computed within- versus between-category locations, especially when there were perceptual cues.

\section{DISCUSSION}

Taken together, the results of this study shed important light on the acquisition of new spatial representations in natural environments. As we predicted, both categorical and fine-grained knowledge increased over time: As students learned more about the specific locations of the campus buildings, they also learned more about which campus region the locations were in (south, middle, or north campus). Both forms of knowledge affected participants' placements of the locations. For example, although upper-class students knew a great deal about the specific configuration of locations of buildings on campus, their judgments were simultaneously more biased than were first-year students', because they had acquired knowledge of the categories on campus. Put another way, participants' placements of the buildings in our experiment (and, presumably, their mental representations of the locations on campus) grew more accurate in terms of fine-grained knowledge within regions - and, thus, for the configuration as a whole - but the placements also became more biased, because of the influence of increasing knowledge about the categories.
The increase in categorical knowledge stems, at least partly, from increasing familiarity with the divisions of the campus, as well as from communication among students. The campus divisions are not, to our knowledge, explicitly marked on any campus maps, but they are referred to frequently in conversations. Thus, they are cognitive and linguistic constructions, and students, therefore, are not likely to learn about the campus divisions simply by navigating on campus. Of course, knowledge gained from navigating around campus would augment knowledge gained from conversations, but it seems unlikely that the categorical knowledge would emerge as it did in the present experiment if students did not hear and learn about the buildings' locational referents and, thus, the campus divisions from others. Thus, the learning and construction of categorical representations of space is both a social and a cognitive process.

The increase in fine-grained knowledge probably stems from an increase in familiarity with the individual building locations, the relations among locations, or both. In other words, there are two (not mutually exclusive) possible explanations for what participants actually learned (and represented) as they learned more about the fine-grained locations of the campus buildings. The first possibility is that students simply acquired more precise knowledge of the specific location of each of the buildings. Thus, through navigating, looking at maps, and using other sources of information, the students increasingly knew more precisely the location of each building. Relational knowledge would emerge as a by-product of this absolute knowledge.

The second possibility is that the participants also learned explicitly about the relations among the locations - that is, they began to code the configuration of locations (e.g., Gentner, 1983; Gentner \& Markman, 1994, 1997; Mou, Zhao, \& McNamara, 2007). Put another way, the participants began to learn where each location was, relative to the other locations. Thus, in contrast to many studies in which participants have learned one location at a time (e.g., Huttenlocher et al., 1991; Spencer \& Hund, 2002; Spencer, Simmering, \& Schutte, 2006), participants in the present experiment learned multiple locations over an extended period of time and, hence, had the possibility of learning about relations among these locations. In addition, the relations among locations could be learned through several means, including maps, navigation, being given directions, taking detours, or learning shortcuts.

Our data do not allow us to distinguish between these two possibilities: Either form of representation could have accounted for the observed results obtained for fine-grained knowledge. However, it is worth noting that knowledge of relations among locations would have the benefit of constraining the location of other buildings, thus making it easier to recall a specific location. Thus, although coding knowledge of relations among locations might be seen as demanding, there is an ultimate payoff in accuracy.

\section{Learning Sequence}

The results also shed light on the time course of learning categorical and fine-grained knowledge. Specifically, 
the results indicate that fine-grained knowledge is learned before categorical knowledge, at least in this case. We found that the influences of categorical knowledge did not emerge (at least statistically) during the first year, whereas fine-grained knowledge did improve across the first three quarters.

This result raises an important question: Is the learning sequence that we have identified (fine-grained before category) generalizable, or is it limited to the particular characteristics of the present study? In our case, the categories are not well defined and, thus, must be learned and cognitively constructed over time. As students progress through their majors, they tend to spend increasingly greater amounts of time on one part of campus (usually at the expense of spending time on the other parts of campus) because of the disciplinary relation among the buildings on each part of campus. Both knowledge of the categorical distinctions and the relevance of these distinctions for where students live and take classes should, therefore, increase with time. We acknowledge, however, that, in other situations, it is possible that the learning sequence might be different. For example, when learning about geographic scale locations (e.g., countries), it is possible that people might learn about categorical divisions (e.g., states or countries) before they learn about specific locations. What is less known, however, is when categorical knowledge about real-world geographical entities at several scales (e.g., navigational, global) begins to bias absolute location estimates (but see Kerkman, Friedman, Brown, Stea, \& Carmichael, 2003). It is also worth noting that Newcombe and Chiang (2007), who trained people extensively on hypothetical geographic spaces (made-up countries within continents) in order to control for learning history, found that biases in participants' judgments differed from those found in real-world geography. They concluded that the biases in the hypothetical spaces were likely to have been based on perceptual heuristics rather than on categorical organization. The important point for the present study is that real-world geography - whether at the geographic or navigable scale - is most typically learned over a much longer period than that used in Newcombe and Chiang. In the present case, it seems to have taken at least a year on campus for the effects of categorization to be revealed in accuracy.

\section{The Influence of Cues on Participants' Placements}

As we expected, we found that the presence of cues (i.e., boundary lines on the map) affected the accuracy of participants' placements. On average, participants' placements were more accurate when there were map cues in the response space. This result is consistent with the work of Kitchin (e.g., Kitchin \& Fotheringham, 1997) and others (e.g., Friedman, 2009), who found that the presence of lines, borders, or other boundaries can influence participants' spatial judgments.

An unexpected finding was that the influence of time on campus (i.e., year in school) on the biases created by category knowledge became evident only when partici- pants responded on the screens with perceptual cuesthat is, the lines that indicated the campus borders. When more experienced participants used the response screen with the eastern and western borders, they tended to show more north-south category bias than did participants with similar levels of experience who did not see the lines on the map.

In interpreting this result, it is important to reiterate that the effect does not represent participants' distorting distances across represented boundaries. The lines that we included were the east and west boundaries of the campus, but participants, nevertheless, showed more bias in the north-south direction when the lines were present than when they were absent. Thus, it cannot be the case that the participants were simply using the lines as boundary markers for the north-, middle-, and south-category boundaries. Instead, the presence of the east and west boundary lines must have cued category knowledge, resulting in participants' placing north- and south-campus locations farther apart than they did when the lines were not present. It is possible that the cues strongly activated the students' knowledge of the campus, which, for them, included the division of the campus into regions. That is, making students think about their campus by seeing the lines that explicitly defined its boundaries may have activated knowledge of the categorical division of the campus, even when these divisions were not explicitly shown.

\section{Implications}

Our results have important implications for at least two lines of research in spatial cognition: the representation and integration of category and fine-grained knowledge and the influences of size or scale on spatial judgments.

The first point concerns the relative contributions of categorical and fine-grained knowledge to judgments within large-scale spaces. Our results help to constrain the category-adjustment model. Huttenlocher et al. (1991) viewed the category-adjustment model as the optimal way to remember locations under conditions of uncertainty. That is, in the original model - even though the categories lead to systematic biases in location estimates - overall, it is assumed that locations at both the category and finegrained knowledge levels are represented accurately and that bias occurs through their Bayesian combination. Even the biased responses are statistically optimal under conditions of uncertainty. If people are unsure of the precise location of a given object or landmark, then augmenting what they do know about the location with category knowledge tends to improve the accuracy of estimates, on average, at recall.

We identified clear effects of both kinds of knowledge and documented that these increase at different rates (Hund \& Plumert, 2002; Huttenlocher et al., 1991). However, the present research, as well as prior studies (e.g., Friedman, 2009; Friedman et al., 2005; Friedman \& Montello, 2006) suggests that the category-adjustment model may not explain performance in large-scale space as well as it does in smaller spaces. One important reason is that the present category-adjustment model has no mechanism 
for learning over the long term, as would be required for estimates in both the current and previous (Friedman, 2009) studies. Indeed, Huttenlocher et al. (2004) showed that participants were insensitive to the distribution of locations in a dot location task; in part, we think that this is because configural knowledge is irrelevant to that task but that it is not irrelevant for remembering real-world locations. Another possibility is simply that the categoryadjustment model does not apply to larger spaces. However, we think that it is more useful to use the present data to constrain the category-adjustment model to account for the results we observed in large space - at both navigable and geographic scales.

In our view, the influence of categories on estimates is not always to make them more accurate, although category and fine-grained information may very well still be combined in a Bayesian manner. The outcome is thus optimal in a Bayesian sense, but the result can be poor if one or more of the priors is inaccurate. This is especially true when beliefs about the location of a category's borders (and thus, its prototype) are wrong by relatively large amounts but the category is still weighted heavily in the judgment. Indeed, beliefs about the location of categories - or about the relations among categoriescan be systematically biased and, hence, can lead to large and systematic errors. It may also be that people trust their categorical knowledge more than their fine-grained knowledge and that they weigh it more heavily for that reason. In the present case, perhaps the establishment of a linguistically and socially constructed category leads to an unusually high salience of categorical information, leading to its overweighting (see Hauser, 2009).

Thus, for example, in Friedman et al. (2005), participants' distorted beliefs about the locations of North American regions in relation to large-scale geographical landmarks led to grossly exaggerated location estimates (and, by inference, distance estimates) between cities across regional borders in Canada, the United States, and Mexico by persons from all three countries. Furthermore, Friedman and Montello (2006) showed that location and distance estimates (as well as "border effects") at a global scale were computed from the same representation; here, we show how that representation changes with time. Likewise, in the present experiment, the effect of the invisible "border" between north and south campus was evident for the upper-level students, but not for the first-year students. The present data, thus, add to the literature on the differences between real-world location estimates and estimates within more abstract "spaces." They particularly address differences between relatively short-term visual memory for dots presented one at a time and longer lasting memories for spatial relations among entities experienced together over much longer time periods (see Friedman, 2009). Thus, the category-adjustment model is constrained, in that it appears to apply primarily to spatial representations in relatively short-term memory; even extensive training on hypothetical maps (Newcombe \& Chiang, 2007) did not produce the kinds of category effects seen with real-world geographic and navigable spaces.

\section{Scale and Research in Spatial Cognition}

Finally, the results are relevant to an ongoing debate regarding the similarities and differences among spaces of vastly different sizes and the psychological processes that operate in them. Several researchers have questioned whether research in small-scale spaces (e.g., computer screens, scale models) is relevant or can be generalized to the much larger spaces in which human spatial cognition and navigation typically occur (e.g., Liben, 2003; Montello, 1993). Our results suggest that research in smalland large-scale spaces can reciprocally inform each other. The category-adjustment model, which was evaluated primarily in small-scale spaces, allowed us to make specific predictions regarding the influence of fine-grained and categorical knowledge on judgments. At the same time, working in large navigable spaces (or geographicalscale spaces) allowed us see the potential limitations of the category-adjustment model. Specifically, whereas the category-adjustment model views the combination of categorical and fine-grained knowledge as resulting in optimal judgments (under conditions of uncertainty), we found that, in larger-scale spaces, biases in judgments about the locations of categories can lead to largely inaccurate absolute judgments. It is worthwhile noting again that our participants never made distance judgments per se; we computed the distances from their absolute location judgments. Thus, irrespective of whether they were encoding only the absolute locations or the relations between buildings, it is clear that, as soon as category-level information was available, it was biased.

In summary, we have shown that both fine-grained and categorical knowledge influence judgments in large-scale, navigable space. Although prior studies (e.g., Hirtle \& Jonides, 1985) have demonstrated that categorical knowledge affects judgments in navigable spaces, to our knowledge, the present data are the first to show how both finegrained and categorical knowledge influence judgments about navigable spaces and when these different forms of representation are learned.

\section{AUTHOR NOTE}

The order of authorship between the first two authors was determined by a flip of a coin. This research was supported by NSF Grant SBE0541957, the Spatial Intelligence and Learning Center, to D.H.U., and a Grant from the Natural Sciences and Engineering Research Council of Canada to A.F. Correspondence should be sent to D. H. Uttal, Department of Psychology, Northwestern University, 2029 Sheridan Road, Evanston, IL 60208-2710 (e-mail: duttal@northwestern.edu) or to A. Friedman, Department of Psychology, University of Alberta, Edmonton, AB, T6G 2E9 Canada.

\section{REFERENCES}

Acredolo, L. P. (1981). Small- and large-scale spatial concepts in infancy and childhood. In L. S. Liben, A. H. Patterson, \& N. Newcombe (Eds.), Spatial representation and behavior across the life span (pp. 63-81). New York: Academic Press.

Carbon, C.-C., \& Leder, H. (2005). The Wall inside the brain: Overestimation of distances crossing the former Iron Curtain. Psychonomic Bulletin \& Review, 12, 746-750.

Cheng, K., Shettleworth, S. J., Huttenlocher, J., \& Rieser, J. J. (2007). Bayesian integration of spatial information. Psychological Bulletin, 133, 625-637.

DeLoache, J. S. (1989). Young children's understanding of the corre- 
spondence between a scale model and a larger space. Cognitive Development, 4, 121-139. doi:10.1016/0885-2014(89)90012-9

DeLoache, J. S. (2000). Dual representation and young children's use of scale models. Child Development, 71, 329-338.

Friedman, A. (2009). The role of categories and spatial cuing in globalscale location estimates. Journal of Experimental Psychology: Learning, Memory, \& Cognition, 35, 94-112. doi:10.1037/a0013590

FriedMAN, A., \& BROWN, N. R. (2000a). Reasoning about geography. Journal of Experimental Psychology: General, 129, 193-219.

FriedMan, A., \& Brown, N. R. (2000b). Updating geographical knowledge: Principles of coherence and inertia. Journal of Experimental Psychology: Learning, Memory, \& Cognition, 26, 900-914.

Friedman, A., Kerkman, D. D., Brown, N. R., Stea, D., \& Cappello, H. M. (2005). Cross-cultural similarities and differences in North Americans' geographic location judgments. Psychonomic Bulletin \& Review, 12, 1054-1060.

Friedman, A., \& KoHLER, B. (2003). Bidimensional regression: Assessing the configural similarity and accuracy of cognitive maps and other two-dimensional data sets. Psychological Methods, 8, 468-491.

Friedman, A., \& Montello, D. R. (2006). Global-scale location and distance estimates: Common representations and strategies in absolute and relative judgments. Journal of Experimental Psychology: Learning, Memory, \& Cognition, 32, 333-346. doi:10.1037/0278 $-7393.32 .3 .333$

GeNTNER, D. (1983). Structure-mapping: A theoretical framework for analogy. Cognitive Science, 7, 155-170.

Gentner, D., \& Markman, A. B. (1994). Structural alignment in comparison: No difference without similarity. Psychological Science, $\mathbf{5}$, 152-158.

Gentner, D., \& Markman, A. B. (1997). Structure mapping in analogy and similarity. American Psychologist, 52, 45-56.

Hauser, M. D. (2009). The possibility of impossible cultures. Nature, 460, 190-196.

Hermer, L., \& Spelke, E. S. (1994). A geometric process for spatial reorientation in young children. Nature, 370, 57-59.

Hermer, L., \& Spelke, E. S. (1996). Modularity and development: The case of spatial reorientation. Cognition, 61, 195-232.

HirTLE, S. C., \& Jonides, J. (1985). Evidence of hierarchies in cognitive maps. Memory \& Cognition, 13, 208-217.

HOLYOAK, K. J., \& MaH, W. A. (1982) Cognitive reference points in judgments of symbolic magnitude. Cognitive Psychology, 14, 328-352.

Hund, A. M., \& Plumert, J. M. (2002). Delay-induced bias in children's memory for location. Child Development, 73, 829-840. doi:10.1111/1467-8624.00441

Hund, A. M., \& PlumerT, J. M. (2003). Does information about what things are influence children's memory for where things are? Developmental Psychology, 39, 939-947.

Hund, A. M., \& Plumert, J. M. (2005). The stability and flexibility of spatial categories. Cognitive Psychology, 50, 1-44.

Huttenlocher, J., Hedges, L. V., Corrigan, B., \& Crawford, L. E. (2004). Spatial categories and the estimation of location. Cognition, 93, 75-97. doi:10.1016/j.cognition.2003.10.006

Huttenlocher, J., Hedges, L. V., \& Duncan, S. (1991). Categories and particulars: Prototype effects in estimating spatial location. Psychological Review, 98, 352-376. doi:10.1037/0033-295X.98.3.352

Huttenlocher, J., Newcombe, N., \& SAnDberg, E. H. (1994). The coding of spatial location in young children. Cognitive Psychology, 27, 115-147. doi:10.1006/cogp.1994.1014

Kerkman, D. D., Friedman, A., Brown, N. R., Stea, D., \& CarMichael, A. (2003). The development of geographic categories and biases. Journal of Experimental Child Psychology, 84, 265-285.

Kitchin, R. M., \& Fotheringham, A. S. (1997). Aggregation issues in cognitive mapping. The Professional Geographer, 49, 269-280.
Learmonth, A. E., Newcombe, N. S., Sheridan, N., \& Jones, M. (2008). Why size counts: Children's spatial reorientation in large and small enclosures. Developmental Science, 11, 414-426.

LiBEN, L. S. (2003). Extending space: Exploring the territory of spatial development. Human Development, 46, 61-68. doi:10.1159/000067777

Loftus, G., \& Masson, M. E. J. (1994). Using confidence intervals in within-subject designs. Psychonomic Bulletin \& Review, 1, 476490.

MAKI, R. H. (1981). Categorization and distance effects with spatial linear orders. Journal of Experimental Psychology: Human Learning \& Memory, 7, 15-32.

MCNAmara, T. P. (1986). Mental representations of spatial relations. Cognitive Psychology, 18, 87-121.

McNamara, T. P., Rump, W., \& Werner, S. (2003). Egocentric and geocentric frames of reference in memory of large-scale space. Psychonomic Bulletin \& Review, 10, 589-595.

MonTEllo, D. R. (1993). Scale and multiple psychologies of space. In A. U. Frank \& I. Campari (Eds.), Spatial information theory: A theoretical basis for GIS (pp. 312-321). Berlin: Springer.

Mou, W., McNamara, T. P., Valiquette, C. M., \& Rump, B. (2004). Allocentric and egocentric updating of spatial memories. Journal of Experimental Psychology: Learning, Memory, \& Cognition, 30, 142-157.

Mou, W., Zhao, M., \& McNamara, T. P. (2007). Layout geometry in the selection of intrinsic frames of reference from multiple viewpoints. Journal of Experimental Psychology: Learning, Memory, \& Cognition, 33, 145-154.

Newcombe, N. S., \& Chiang, N. C.-R. (2007). Learning geographical information from hypothetical maps. Memory \& Cognition, 35, 895-909.

Newcombe, N., \& Liben, L. S. (1982). Barrier effects in the cognitive maps of children and adults. Journal of Experimental Child Psychology, 34, 46-58.

Plumert, J. M., \& Hund, A. M. (2001). The development of memory for location: What role do spatial prototypes play? Child Development, 72, 370-384. doi:10.1111/1467-8624.00284

Simmering, V. R., \& SPencer, J. P. (2007). Carving up space at imaginary joints: Can people mentally impose arbitrary spatial category boundaries? Journal of Experimental Psychology: Human Perception \& Performance, 33, 871-894. doi:10.1037/0096-1523.33.4.871

SPENCER, J. P., \& Hund, A. M. (2002). Prototypes and particulars: Geometric and experience-dependent spatial categories. Journal of Experimental Psychology: General, 131, 16-37. doi:10.1037/0096 $-3445.131 .1 .16$

Spencer, J. P., Simmering, V. R., \& Schutte, A. R. (2006). Toward a formal theory of flexible spatial behavior: Geometric category biases generalize across pointing and verbal response types. Journal of Experimental Psychology: Human Perception \& Performance, 32, 473-490.

Stevens, A., \& Coupe, P. (1978). Distortions in judged spatial relations. Cognitive Psychology, 10, 422-437.

Tobler, W. R. (1965). Computation of the correspondence of geographical patterns. Papers of the Regional Science Association, 15, 131-139.

Tobler, W. R. (1994). Bidimensional regression. Geographical Analysis, 26, 187-212

Waller, D., Montello, D. R., Richardson, A. E., \& Hegarty, M. (2002). Orientation specificity and spatial updating of memories for layouts. Journal of Experimental Psychology, Learning, Memory, \& Cognition, 28, 1051-1063.

WENDEROTH, P., \& VAN DER ZWAN, R. (1991). Local and global mechanisms of one- and two-dimensional orientation illusions. Perception \& Psychophysics, 50, 321-332. 


\section{APPENDIX}

Each participant's reconstruction of the campus was fit to the actual location, as represented on the map shown in Figure 1. The four BDR parameters derived from the estimates reflect how each individual's cognitive map locations would have to be scaled, rotated, and translated north-south and east-west to best fit the actual campus map (see Friedman \& Kohler, 2003, for details). It is not necessarily clear how these parameters map onto either fine-grained or category knowledge; it is possible that these parameters would be part of both levels of representation. That is, because the same parameters apply to each location, they can reflect (or be reflected in) fine-grained information about each location individually, as well as information about biases or distortions in the configuration as a whole that might be either fine-grained or inherited from categorical knowledge (e.g., does the configuration need to be scaled, rotated, or translated east-west or north-south). Consequently, we did not make a priori predictions about these parameters, other than to suppose that, if they reflected fine-grained knowledge, they should improve as a function of time. In addition, as with the BDR coefficient, the group with perceptual cues should be more accurate (for each parameter) than the group without cues would be. The means for each parameter are shown as a function of class and cue group in Table A1. We discuss each in turn.

Table A1

Average Bidimensional Regression (BDR) Parameters for Scale, Rotation, and Translation As a Function of Class and Cue Group BDR Parameters

\begin{tabular}{lrrrr}
\hline & \multicolumn{4}{c}{ BDR Parameters } \\
\cline { 2 - 5 } & & & \multicolumn{2}{c}{ Translation (Pixels) } \\
\cline { 4 - 5 } Class & Scale $^{*}$ & Rotation & North-South & East-West \\
\hline First year & & & & \\
$\quad$ Cues & .917 & $-3.28^{\circ}$ & 198.85 & 10.13 \\
$\quad$ No cues & 1.047 & $18.83^{\circ}$ & 116.19 & 7.15 \\
Upper level & & & & -84.59 \\
$\quad$ Cues & 1.022 & $-1.19^{\circ}$ & 52.47 & -94.41 \\
$\quad$ No cues & 1.030 & $19.86^{\circ}$ & 136.15 & \\
\hline
\end{tabular}

*The scale parameter is a multiplier (how much each estimated $x, y$ value would have to be multiplied by to best fit into the real map). For the rotation parameter, negative angles are clockwise rotations. For the translation parameters, because the 0,0 point is at the top left corner of the screen, larger values imply locations that are farther south and east. As an example, to best fit the average cognitive map of the first-year students who had the cues, one would take each estimated $x$ and $y$ value and multiply it by 0.917 (to decrease the scale by around $10 \%$ ), rotate it clockwise by $3.28^{\circ}$, add 200 pixels to the $y$ value (to move it south), and add 10.13 pixels to the $x$ value (to move it east).

\section{Scale}

The scale parameter indicates how much the estimated locations (i.e., the cognitive map) have to be expanded or shrunk to best fit the actual configuration of tested campus buildings. For the first-year students, there were main effects of cue $\left[F(1,87)=6.41, M S_{\mathrm{e}}=0.08, \eta_{\mathrm{p}}^{2}=.069\right]$ and quarter $\left[F(2,87)=5.49, M S_{\mathrm{e}}=0.08, \eta_{\mathrm{p}}^{2}=\right.$ $.112]$, and there was an interaction between the two variables $\left[F(2,87)=5.11, M S_{\mathrm{e}}=0.08, \eta_{\mathrm{p}}^{2}=.105\right.$; see Table A2]. Essentially, the presence of perceptual cues kept the first-year students on a relatively correct scale, whereas, without cues, the expressed cognitive maps increased in scale with time. Presumably, the buildings were moving "farther apart" in participants' cognitive representations, even as knowledge about the configuration among the buildings' locations increased in accuracy. There is certainly some precedence in the spatial memory literature for increasing distance between locations as a function of familiarity (Holyoak \& Mah, 1982).

Table A2

Changes in the Scale of the Cognitive Maps for First-Year Students As a Function of Cue Group and Quarter

\begin{tabular}{lccc}
\hline Condition & First Quarter & Second Quarter & Third Quarter \\
\hline Cues & 0.905 & 0.849 & 0.986 \\
No cues & 0.814 & 1.198 & 1.182 \\
\hline
\end{tabular}

In contrast to the first-year students, there were no effects of scale as a function of cue group for the upperlevel students. The average scale parameter was 1.02 for the cue group, and it was 1.03 for the no-cue group. There were also no effects of scale as a function either of cue group or of class in the combined analysis of both first-year and upper-level students. Thus, by the end of the first year, participants who saw the perceptual cues responded at the accurate scale, whereas those who did not have perceptual cues used a scale that was about 


\section{APPENDIX (Continued)}

$20 \%$ larger than it should have been. After the first year, participants appeared to have accurate knowledge of the campus' scale with or without perceptual cues.

\section{Rotation}

In the analysis of the rotation parameter with all participants included, there was a main effect of cue group $\left[F(1,138)=6.64, M S_{\mathrm{e}}=2,200.72, \eta_{\mathrm{p}}^{2}=.046\right]$. There did not appear to be an increase in accuracy with time for this parameter, because there was not a significant effect of either quarter for the first-year students or of class in the combined analysis. There was almost no angular difference between the actual and cognitive maps for participants of all ages in the cue group $\left(-2.2^{\circ}\right)$, whereas participants who did not have the cues had rotated the actual map's coordinates by approximately $19.3^{\circ}$ counterclockwise. These results are similar to Kitchin and Fotheringham's (1997) and Friedman's (2009) findings that providing perceptual cues such as borders improves accuracy, at least in part because the cues help participants align their responses properly.

\section{East-West Translation}

There were no significant effects in any of the analyses for the east-west translation parameter.

\section{North-South Translation}

For the first-year students, there was a main effect of quarter $\left[F(2,87)=6.44, M S_{\mathrm{e}}=213,534.10, \eta_{\mathrm{p}}^{2}=.129\right]$. The campus started out too far to the north and moved significantly southward as a whole by the end of the first year. There were no other significant effects for this parameter in that analysis, nor were there significant effects in the combined analysis. 